\title{
THE SPATIAL DISTRIBUTION OF OH AND CN RADICALS IN THE COMA OF COMET ENCKE
}

\author{
Chandrasiri A. Ihalawela ${ }^{1,3}$, Donna M. Pierce ${ }^{1}$, Garrett R. Dorman ${ }^{1}$, and Anita L. Cochran ${ }^{2}$ \\ ${ }^{1}$ Department of Physics and Astronomy, Mississippi State University, P.O. Box 5167, Mississippi State, MS 39762-5167, USA; \\ cai11@msstate.edu, ci856509@ohio.edu,dmp149@msstate.edu,grd33@msstate.edu \\ ${ }^{2}$ McDonald Observatory, University of Texas at Austin, 1 University Station, C1402, Austin, TX 78712-0259, USA; \\ anita@barolo.as.utexas.edu \\ Received 2010 September 11; accepted 2011 August 4; published 2011 October 21
}

\begin{abstract}
Multiple potential parent species have been proposed to explain $\mathrm{CN}$ abundances in comet comae, but the parent has not been definitively identified for all comets. This study examines the spatial distribution of $\mathrm{CN}$ radicals in the coma of comet Encke and determines the likelihood that $\mathrm{CN}$ is a photodissociative daughter of $\mathrm{HCN}$ in the coma. Comet Encke is the shortest orbital period (3.3 years) comet known and also has a low dust-to-gas ratio based on optical observations. Observations of CN were obtained from 2003 October 22 to 24 , using the $2.7 \mathrm{~m}$ telescope at McDonald Observatory. To determine the parent of $\mathrm{CN}$, the classical vectorial model was modified by using a cone shape in order to reproduce Encke's highly aspherical and asymmetric coma. To test the robustness of the modified model, the spatial distribution of $\mathrm{OH}$ was also modeled. This also allowed us to obtain $\mathrm{CN} / \mathrm{OH}$ ratios in the coma. Overall, we find the $\mathrm{CN} / \mathrm{OH}$ ratio to be $0.009 \pm 0.004$. The results are consistent with $\mathrm{HCN}$ being the photodissociative parent of $\mathrm{CN}$, but we cannot completely rule out other possible parents such as $\mathrm{CH}_{3} \mathrm{CN}$ and $\mathrm{HC}_{3} \mathrm{~N}$. We also found that the fan-like feature spans $\sim 90^{\circ}$, consistent with the results of Woodney et al..
\end{abstract}

Key words: astrochemistry - comets: general - comets: individual (Encke) - molecular processes

Online-only material: color figures

\section{INTRODUCTION}

Comets are both physically and chemically diverse due to the variation of temperatures that their constituent icy grains would have experienced over a wide range of distances in the outer solar nebula prior to being incorporated into the nucleus (A'Hearn et al. 1995). Therefore, cometary nuclei play a significant role in solar system studies by preserving in their interiors records of the conditions present at the time and place of their formation. However, the coma obscures the nucleus from view when observations are most easily performed. Thus, it is important to understand the nature of cometary comae in order to understand the nature of cometary nuclei.

The $\mathrm{CN}$ radical was one of the first species detected in cometary comae, and it has one of the strongest spectral signatures after $\mathrm{OH}$. CN gas jets were first observed in $1 \mathrm{P} /$ Halley, thus demonstrating the possibility of pure gas jets existing in cometary comae (A'Hearn et al. 1986a, 1986b). The violet system $\left(B^{2} \Sigma^{+}-X^{2} \Sigma^{+}\right)$and the red system $\left(A^{2} \Pi-X^{2} \Sigma^{+}\right)$are two electronic band systems of $\mathrm{CN}$ that can be observed in optical cometary spectra (Feldman et al. 2004). However, the origin of the $\mathrm{CN}$ radical in comets is still not fully understood, and there are multiple potential parent species. HCN is the most widely suggested parent (Schloerb et al. 1987; Ziurys et al. 1999). HCN is now routinely observed at infrared and millimeter wavelengths (Biver et al. 2002; DiSanti \& Mumma 2008; Dello Russo et al. 2009), and HCN is generally used in the millimeter as the standard molecule against which to measure abundances of other molecules, just as $\mathrm{H}_{2} \mathrm{O}$ is used for measuring abundances at infrared wavelengths. Woodney et al. (2002) concluded from the C/1995 O1 (Hale-Bopp) apparition that $\mathrm{HCN}$ is the primary parent, even though there is a discrepancy between the HCN destruction scale length and the $\mathrm{CN}$

\footnotetext{
3 Current address: Physics and Astronomy Department, Ohio University, Clippinger Labs, Athens, OH 45701-2979, USA.
}

production scale length. Inconsistency of the $\mathrm{CN}$-parent scale length with $\mathrm{HCN}$ photodissociation suggests that there may be multiple candidates for the $\mathrm{CN}$ parent (Bockelée-Morvan et al. 1984; Bockelée-Morvan \& Crovisier 1985). Other studies suggest that $\mathrm{HCN}$ could be the sole parent of $\mathrm{CN}$ for heliocentric distances larger than $3 \mathrm{AU}$, and that one or more additional parents are required to explain $\mathrm{CN}$ abundances for heliocentric distances less than $3 \mathrm{AU}$ (Fray et al. 2005). However, Combi \& Delsemme (1980b) and Cochran (1982) showed complete agreement of the $\mathrm{CN}$-parent and $\mathrm{HCN}$ scale lengths according to standard photodissociation models. HCN was first detected in comet Kohoutek (Huebner et al. 1974), and the presence of $\mathrm{HCN}$ in comets was further confirmed from observations of the 1P/Halley apparition in 1986 (Schloerb et al. 1986; Despois et al. 1986; Winnberg et al. 1987; Bockelée-Morvan et al. 1987). Excluding $\mathrm{HCN}$, possible parents for $\mathrm{CN}$ include CHON particles, $\mathrm{CH}_{3} \mathrm{CN}, \mathrm{HC}_{3} \mathrm{~N}, \mathrm{HNC}$, and $\mathrm{C}_{2} \mathrm{~N}_{2}$. Bockelée-Morvan \& Crovisier (1985) and Festou et al. (1998) have suggested the possibility of $\mathrm{C}_{2} \mathrm{~N}_{2}$, as well as $\mathrm{HC}_{3} \mathrm{~N}$ (Bockelée-Morvan \& Crovisier 1985). Krasnopolsky (1991) further supported the $\mathrm{HC}_{3} \mathrm{~N}$ contribution for $\mathrm{CN}$ and proposed another parent: $\mathrm{C}_{4} \mathrm{~N}_{2}$. The consistency of parent scale length and production rate of $\mathrm{CN}$ with the observations and the ability to produce the highly collimated jets observed in some comets are key selection factors for the $\mathrm{CN}$ parent (Woodney et al. 2002).

Comet 2P/Encke has the shortest known cometary orbital period, just 3.3 years. Encke is a small, dark, dust-poor, and asymmetrically outgassing comet. Newburn \& Spinrad (1985) categorized Encke as a dust-poor comet after analyzing the optical spectra of 17 comets, and Gehrz et al. (1989) classified Encke as an IR (Infrared) type 1, i.e., gas-rich and dust-poor. $\operatorname{Af} \rho$, a measurement of production of dust in comets, is very small for Encke $\left[\mathrm{Af} \rho / Q(\mathrm{OH})=4.27 \times 10^{-27}\right]$ as compared to the mean of the sampled population $\left[\mathrm{Af} \rho / Q(\mathrm{OH})=1.51 \times 10^{-26}\right]$ (A'Hearn et al. 1984, 1995). Infrared Space Observatory 
Table 1

Times of Comet Observations

\begin{tabular}{lcc}
\hline \hline Observation Details & Position Angles & Time Range (UT) \\
\hline 2003 Oct 22 & $90^{\circ}, 270^{\circ}$ & $3: 22-4: 46$ \\
$R=1.40 \mathrm{AU} ; \Delta=0.45 \mathrm{AU}$ & $0^{\circ}, 180^{\circ}$ & $4: 54-6: 10$ \\
& $150^{\circ}, 330^{\circ}$ & $6: 23-6: 53$ \\
& $120^{\circ}, 300^{\circ}$ & $7: 51-8: 56$ \\
& $60^{\circ}, 240^{\circ}$ & $9: 13-10: 16$ \\
& $30^{\circ}, 210^{\circ}$ & $10: 28-11: 33$ \\
\hline 2003 Oct 23 & $90^{\circ}, 270^{\circ}$ & $3: 01-4: 07$ \\
& $165^{\circ}, 345^{\circ}$ & $4: 51-5: 21$ \\
& $0^{\circ}, 180^{\circ}$ & $5: 34-6: 38$ \\
& $15^{\circ}, 195^{\circ}$ & $6: 44-7: 52$ \\
& $150^{\circ}, 330^{\circ}$ & $8: 00-9: 07$ \\
& $135^{\circ}, 315^{\circ}$ & $9: 15-10: 18$ \\
& $120^{\circ}, 300^{\circ}$ & $10: 25-11: 27$ \\
\hline$=0.44 \mathrm{AU}$ & $90^{\circ}, 270^{\circ}$ & $2: 57-4: 03$ \\
& $0^{\circ}, 180^{\circ}$ & $4: 17-5: 20$ \\
& $70^{\circ}, 250^{\circ}$ & $5: 33-6: 41$ \\
& $45^{\circ}, 225^{\circ}$ & $6: 55-8: 02$ \\
& $160^{\circ}, 340^{\circ}$ & $8: 13-9: 23$ \\
& $135^{\circ}, 315^{\circ}$ & $9: 31-10: 39$ \\
& $150^{\circ}, 330^{\circ}$ & $10: 45-11: 51$ \\
\hline
\end{tabular}

observations of Encke's dust trail by Reach et al. (2000) and Lisse et al. (2004) show that the dust/gas ratio (by mass) is in the range of 10-30 and dominated by large $(>20 \mu \mathrm{m})$ grains, accounting for the classification of Encke as a gas-rich comet in the optical. The low gas-to-dust ratio of Encke makes it a suitable object on which to test models of photochemistry in order to identify parent molecules of many radical species in comets, including $\mathrm{CN}$, without having to account for significant contributions of these molecules from small (sub- $\mu \mathrm{m}$ sized) dust grains (e.g., CHON particles).

According to Sekanina (1987), asymmetric outgassing is observed in comets due to collimated ejecta from discrete active areas on the sunlit side of rotating cometary nuclei. These asymmetric outgassing structures can be spiral jets, semicircular halos, straight plumes, sharp spikes, snail-shell-like features, and fans with or without jets. Examining the apparitions of Encke from 1924 to 1984, Sekanina (1988) determined the orientation of Encke's prominent sunward-facing fan, which is seen on every apparition, by identifying two discrete emission sources in the northern and southern hemispheres with areas and latitudes of 0.4 and $0.6 \mathrm{~km}^{2}$ and $+55^{\circ}$ and $-75^{\circ}$, respectively. This result was confirmed by Festou \& Barale (2000). However, Woodney et al. (2007) have further suggested the possibility of a single fan with an angle of $90^{\circ}$.

\section{OBSERVATIONS}

Observations of comet Encke were conducted from 2003 October 22 to 24 , using the $2.7 \mathrm{~m}$ telescope $(f / 17.7)$ at McDonald Observatory. The spectral range for the observations was 3000-5600 $\AA$ at a resolving power of 500. The spatial scale and slit dimensions were $1.28 \operatorname{arcsec}_{\text {pixel }}{ }^{-1}$ and $2 \operatorname{arcsec} \times$ 150 arcsec, respectively. For each night, the center of the slit was placed over the nucleus in multiple position angle orientations in order to map the spatial distribution of all observed species. Two position angles were sampled with each observation, $180^{\circ}$ opposite each other. Table 1 lists the times of comet observations for the different position angles (two $1800 \mathrm{~s}$ observations were taken during the specified time frame for each position angle).
The species with transitions falling in the bandpass were $\mathrm{CN}, \mathrm{C}_{2}, \mathrm{C}_{3}, \mathrm{NH}$, and $\mathrm{OH}$. The raw spectra were prepared for analysis by performing bias correction, flat fielding, wavelength, and flux calibration. Then, the sky spectrum (5577 $\AA$ night sky $\mathrm{O}\left({ }^{1} \mathrm{~S}\right)$ line) was subtracted from the observed comet spectrum (Cochran et al. 2009). The (weak) solar continuum spectrum was removed by flux weighting solar analog stars. This was achieved by measuring the average flux at up to 11 continuum regions for both the comet and solar analog spectra. These fluxes were ratioed, and a spline was fit to the ratios to give a multiplicative factor for the solar spectrum so that it is the same color as the comet. This color-corrected solar spectrum was then removed from the cometary spectrum prior to measuring the remaining gas signature. Finally, integrated fluxes were converted into column densities using standard fluorescence efficiencies ( $g$-factors) for $\mathrm{C}_{2}$ and $\mathrm{C}_{3}$ (Cochran et al. 1992, 2009). Swings effect calculations were used to derive $g$-factors for OH (Schleicher \& A'Hearn 1988), NH (Kim et al. 1989), and CN (Tatum 1984).

Plots of column densities of $\mathrm{CN}$ and $\mathrm{OH}$ with respect to the radial distance from the nucleus for each position angle sampled on the first night of observations are shown in Figure 1. Contour maps of the spatial distribution of $\mathrm{CN}$ for each night were constructed from column density profiles as shown in Figure 2.

\section{THE VECTORIAL MODEL}

Spherical symmetry of the coma is a classical assumption in the original vectorial model (Festou 1981), which contributes to its computational simplicity. However, an aspherical and asymmetric coma is necessary to explain comet Encke's prominent sunward-facing fan. Therefore, we have modified the vectorial model to accommodate Encke's highly asymmetric coma. The model begins its calculations in the same manner as the original vectorial model, but it performs the line-of-sight integration of the density distribution of the species of interest through a solid cone instead of a sphere. For a given cone angle $(\Theta)$, the generalized impact parameter, $b$, is defined with respect to the line of sight through the coma (Figure 3 shows the line of sight as it cuts through the fan). Furthermore, $\Psi$ and $\Phi$ are the phase angle and the angle between $b$ and the symmetry axis of the fan, respectively. Because the model requires information on the viewing geometry through the specific region of interest in the coma, the profile generated by the model is only valid for a single position angle. To reproduce the entire coma, the model must be given new input parameters for each different position angle.

\section{RESULTS AND DISCUSSION}

The inclination angle of the generalized impact parameter $\Phi$ was found to be $\sim 63^{\circ}$, based on the phase angle, $\Psi \sim 27^{\circ}$ $\left(\Psi=90^{\circ}-\Phi\right)$, in order to incorporate the viewing geometry from the Earth. The model was first used to reproduce the observed $\mathrm{OH}$ profiles, which also served as a test of the model's ability to reproduce the profiles for multiple position angles. Figure 4 shows $\mathrm{OH}$ observations for several different position angles sampled on 2003 October 23. Two sets of data were obtained for each position angle, and both are shown in the figures, along with the best-fit model. Model fits to the $\mathrm{OH}$ profiles were used to determine the cone angle $\Theta$. Best fits yielded $\Theta=45^{\circ}$.

The model was next applied to the observed $\mathrm{CN}$ profiles. Analysis was done for each sunward and anti-sunward position 

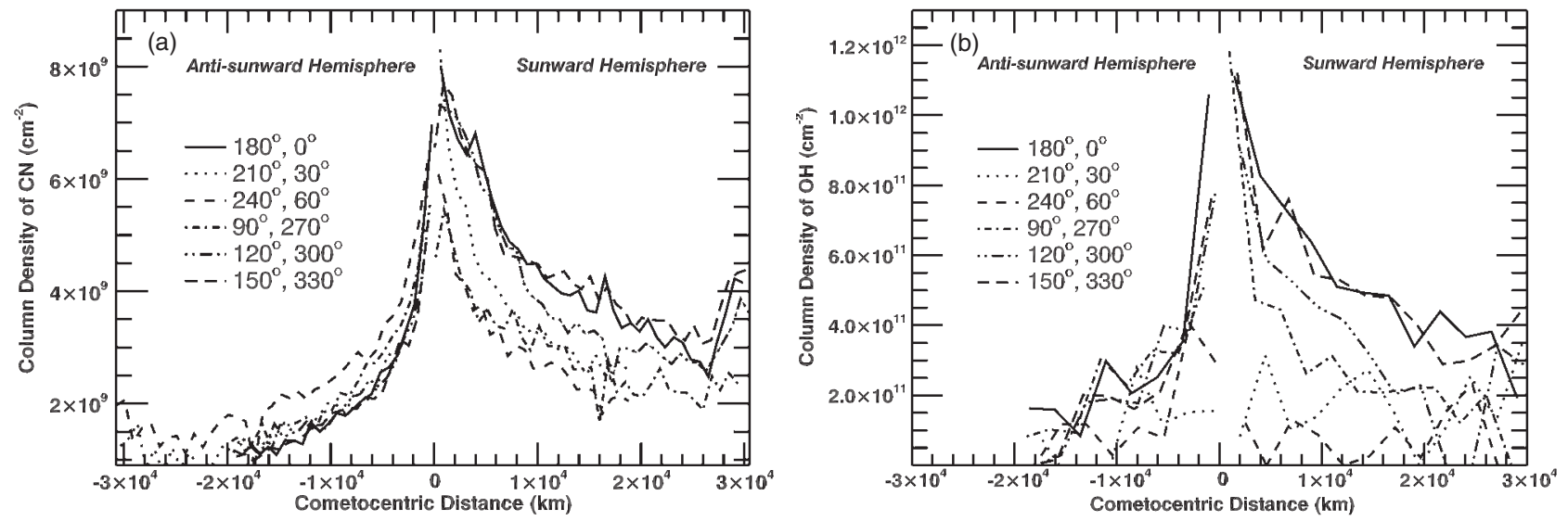

Figure 1. Densities of (a) $\mathrm{CN}$ and (b) $\mathrm{OH}$ with respect to cometocentric distance for all sampled position angles observed on 2003 October 22 . The sampled position angles containing the sunward-facing hemisphere are plotted with positive values of cometocentric distance. The anti-sunward hemisphere is plotted with negative distances. In the legends, the position angles mentioned first have negative cometocentric distances.
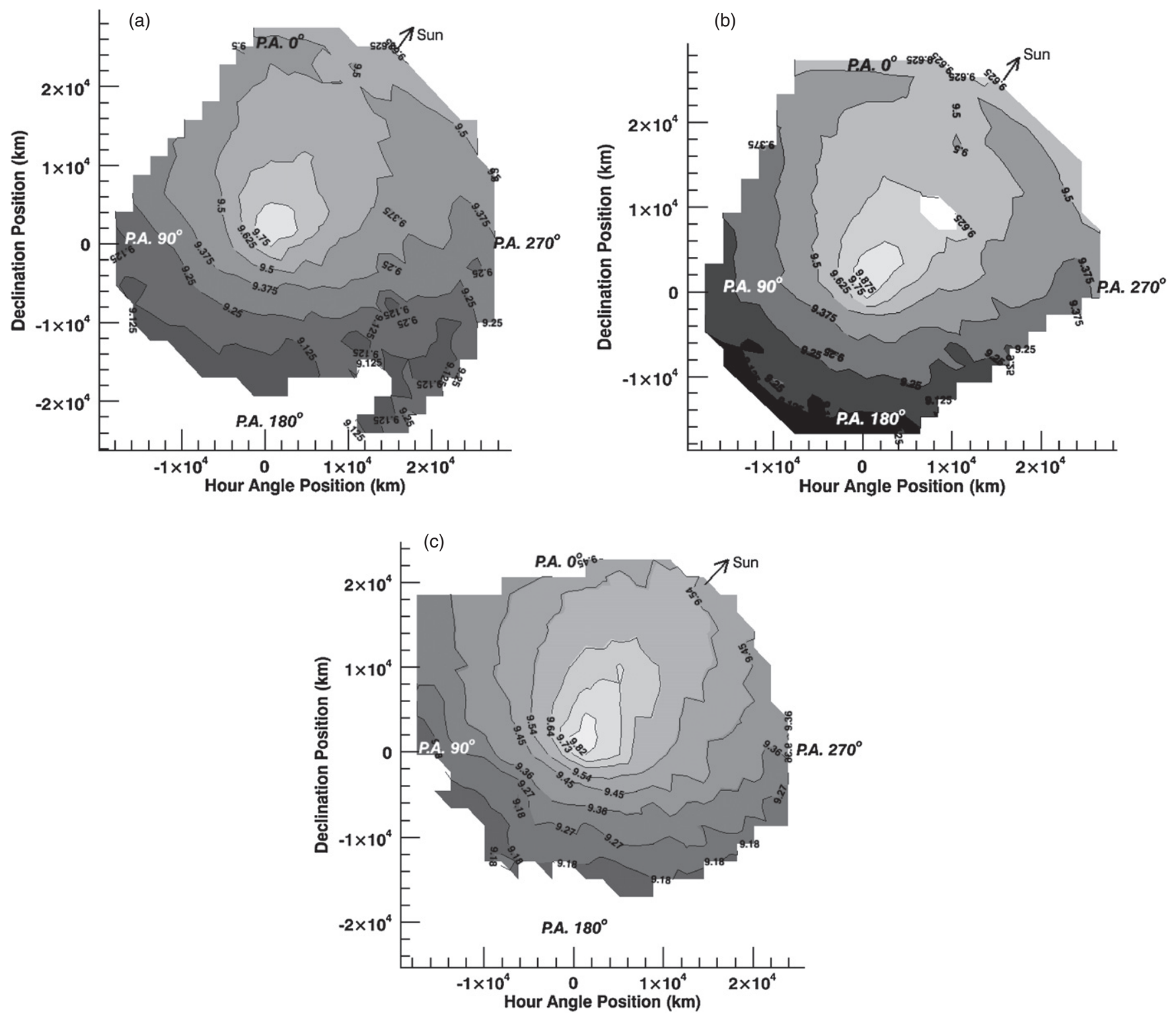

Figure 2. Maps of CN derived from the observed CN emission for 2003 October (a) 22, (b) 23, and (c) 24. Lighter shades indicate higher column densities (given as $\log \mathrm{cm}^{-2}$ ). The position angles of $0^{\circ}, 90^{\circ}, 180^{\circ}$, and $270^{\circ}$ are indicated on each map. Cometocentric profiles of $\mathrm{CN}$ along several position angles of the October 22 map are shown in Figure 1. 


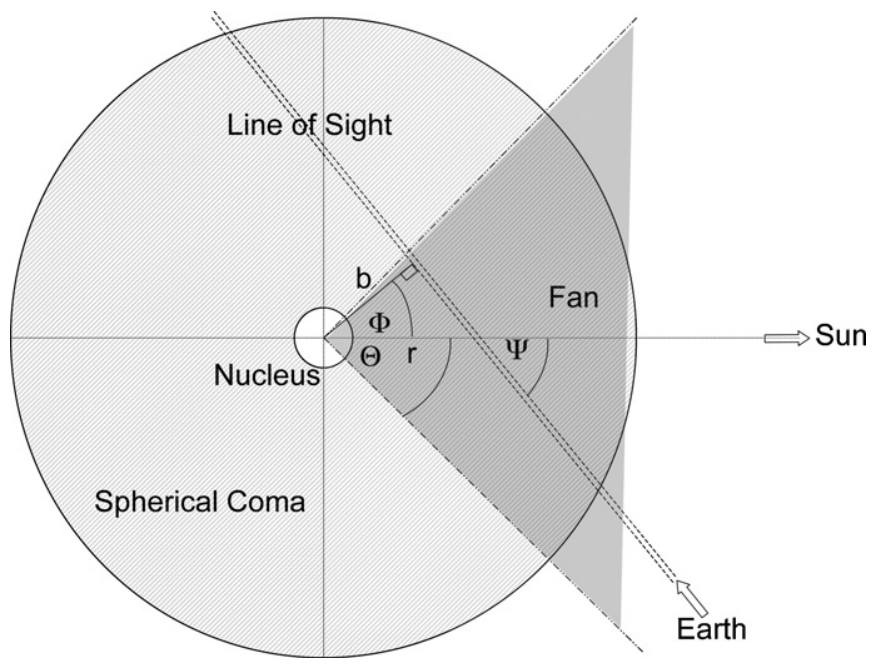

Figure 3. Schematic diagram of the line-of-sight integration through the coneshaped coma model used to compare with the observations, incorporating the viewing geometry. The classical spherical coma is also shown for comparison. The central axis of the fan corresponds to the position angle $330^{\circ}$.

angle within the coma. The photodissociation rates used for $\mathrm{HCN}$ and $\mathrm{CN}$ are $1.33 \times 10^{-5} \mathrm{~s}^{-1}$ and $5.0 \times 10^{-6} \mathrm{~s}^{-1}$, respectively (Huebner et al. 1992; Crovisier 1994). For examinations of $\mathrm{OH}$ profiles, the photodissociation rates of $\mathrm{H}_{2} \mathrm{O}$ and $\mathrm{OH}$ were $1.38 \times 10^{-5} \mathrm{~s}^{-1}$ and $1.1 \times 10^{-5} \mathrm{~s}^{-1}$, respectively (Huebner et al. 1992; Crovisier 1994). Figures 5-7 show the best-fit models for each of the sampled position angles for all three nights. The single-cone model does a reasonable job of reproducing the coma as a whole. The primary difference between the sunward and anti-sunward side is the line of sight through the cone. The sunward position angles are viewed along lines of sight that pass through the central region of the cone, while anti-sunward position angles are viewed along lines of sight that graze the outer rim of the cone.

In general, the model reasonably reproduces the observed $\mathrm{OH}$ and $\mathrm{CN}$ profiles. However, some discrepancies were found for some of the sampled position angles, most likely due to model limitations and observational circumstances. The inability of the model to reproduce strong non-radial influences, such as acceleration of the gas or other corkscrew behavior, is the major limitation of the vectorial model. Therefore, any curling of the fan due to non-radial motion or nucleus rotation is not included in the profiles generated by the model. Additionally, some discrepancies between the model profiles and the observations were seen in the inner coma for position angles closer to the center of the fan. This could be due to optical depth (not included in our model), which can prematurely lower column density determinations close to the nucleus, or this could be due to seeing and guiding effects, although this should similarly affect the $\mathrm{OH}$ profiles. Acceleration of the gas in the inner coma could also affect the observed profiles. We also note some deviations of the model from the observations for larger radial distances $\left(3 \times 10^{4} \mathrm{~km}\right.$ and beyond), which may be due to solar wind interaction with the coma. Overall, the model reasonably reproduces the observations beyond the collisional zone $(\sim 500 \mathrm{~km})$.

The presence of HCN in interstellar clouds and Titan's atmosphere, the detection of $\mathrm{HCN}$ in comet Kohoutek and its measurement in most recent comets, and the agreement of $\mathrm{CN}$ parent and $\mathrm{HCN}$ scale lengths are reasons cited for acceptance of $\mathrm{HCN}$ as the parent of CN (Bockelée-Morvan \& Crovisier 1985). Although the consistency in scale lengths (in Encke)
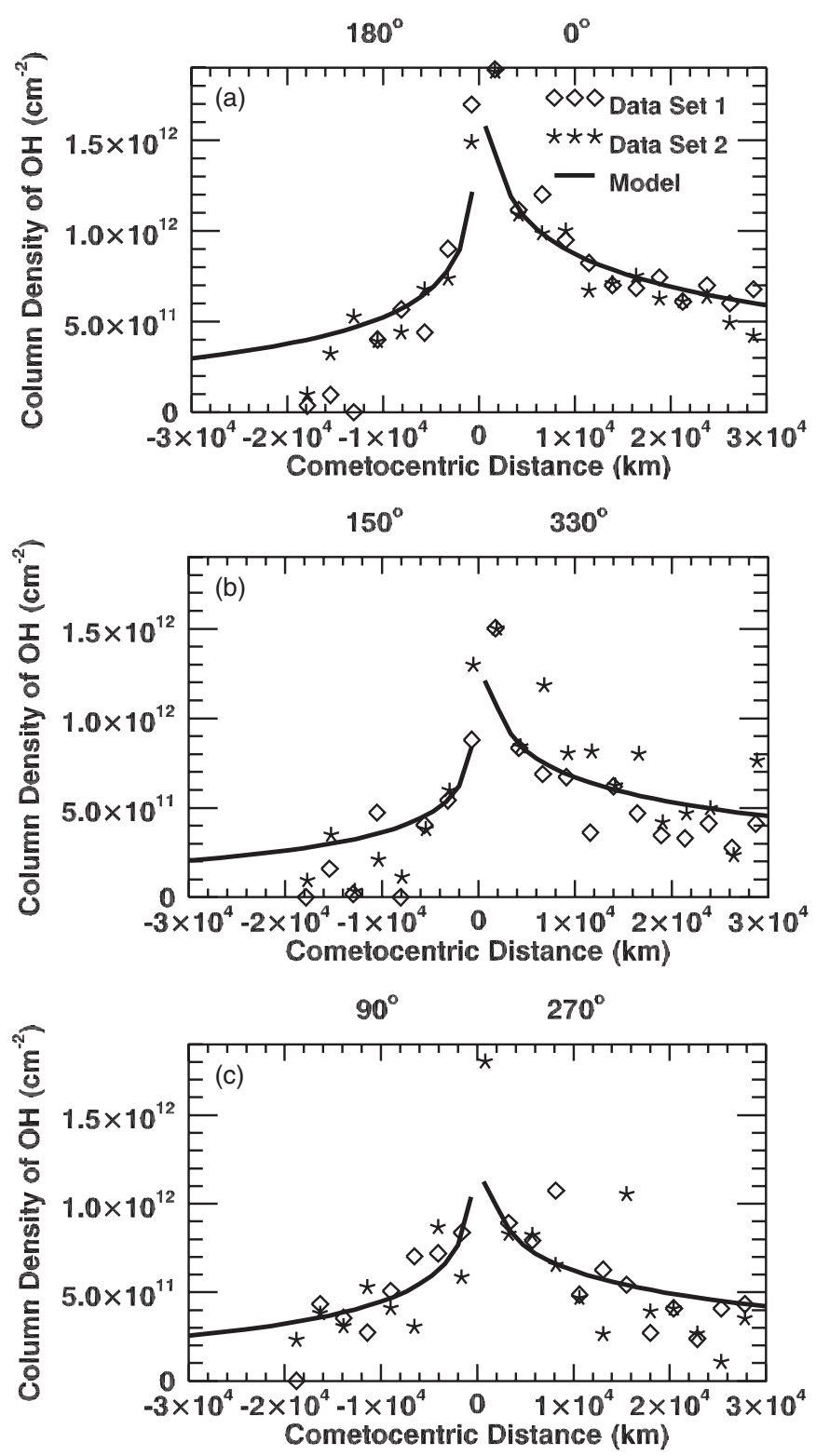

Figure 4. Comparison of best-fit model results with observations of $\mathrm{OH}$ for 2003 October 23 , showing position angles: (a) $0^{\circ}$ and $180^{\circ}$, (b) $150^{\circ}$ and $330^{\circ}$, and (c) $90^{\circ}$ and $270^{\circ}$. In each case, the sampled position angles containing the fan are plotted with positive distances, while anti-sunward position angles are plotted with negative distances.

between $\mathrm{HCN}$ and the required $\mathrm{CN}$ parent is suggestive of this as well, a quantitative comparison of production rates in a larger sample of comets is needed to confirm this relationship. However, other possible parents of $\mathrm{CN}$ have been suggested due to $\mathrm{HCN}$ and $\mathrm{CN}$ production rate discrepancies in visible spectroscopic observations for some comets (Bockelée-Morvan et al. 1984). Bockelée-Morvan \& Crovisier (1985) also showed that $\mathrm{HCN}$ and $\mathrm{CH}_{3} \mathrm{CN}$ are inconsistent with hydrodynamical models due to slow coma expansion. Other potential parent species such as $\mathrm{C}_{2} \mathrm{~N}_{2}, \mathrm{HC}_{3} \mathrm{~N}$, and $\mathrm{CHON}$ particles have been suggested as the $\mathrm{CN}$ parent to explain the observed $\mathrm{CN}$ jets in 1P/Halley (A'Hearn et al. 1986a). Festou et al. (1998) found that the best $\mathrm{CN}$-parent lifetime with typical velocities was $\mathrm{C}_{2} \mathrm{~N}_{2}$. Bonev \& Komitov (2000) examined the Haser scale lengths of potential $\mathrm{CN}$ parents and concluded that the observed brightness distribution of $\mathrm{CN}$ could be explained with a sole parent of $\mathrm{C}_{2} \mathrm{~N}_{2}$. 

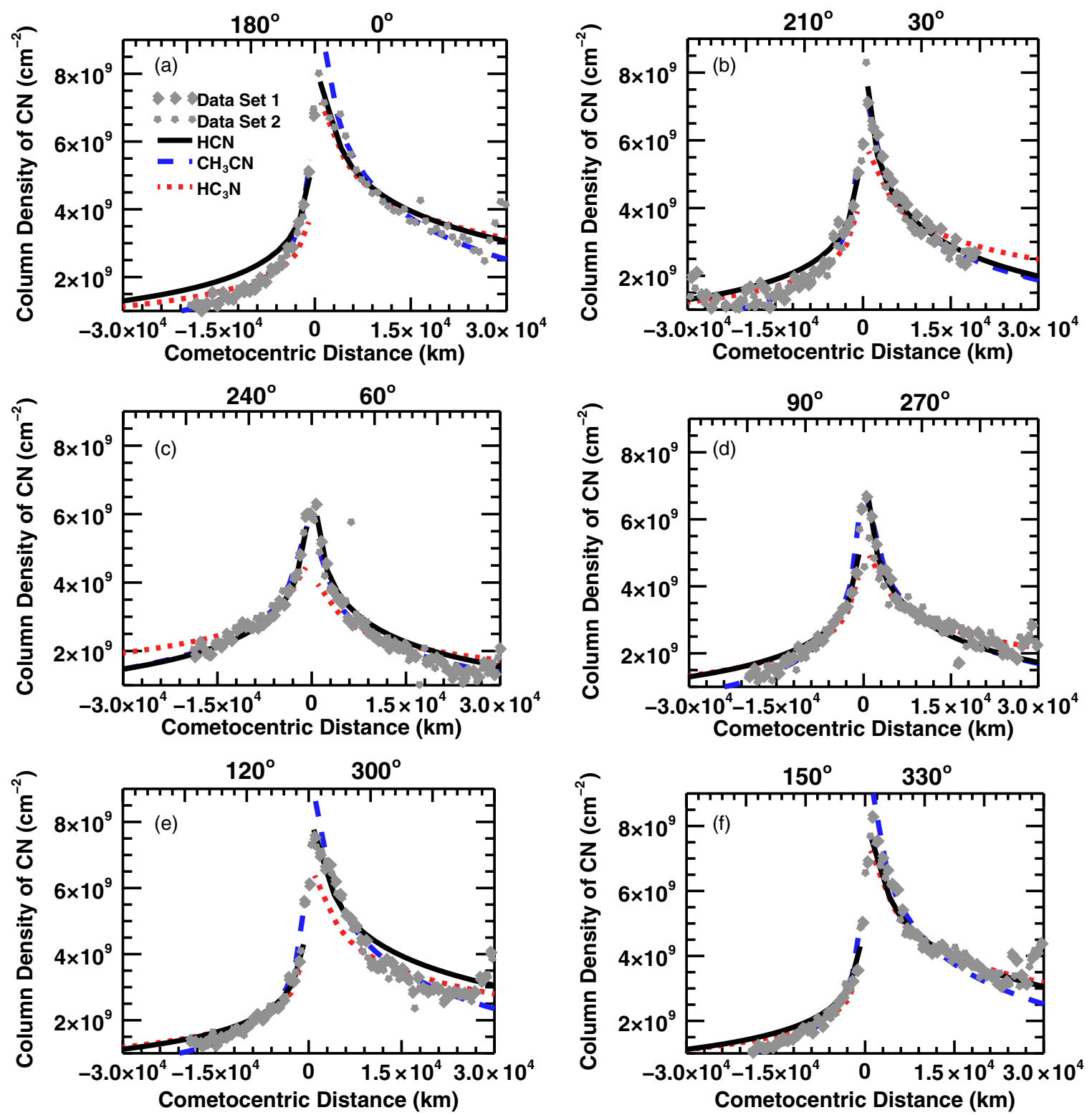

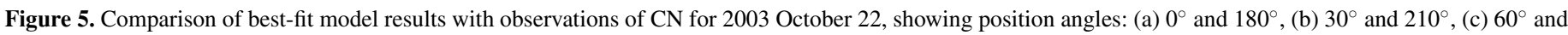

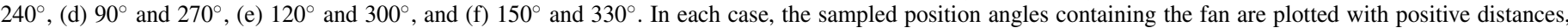

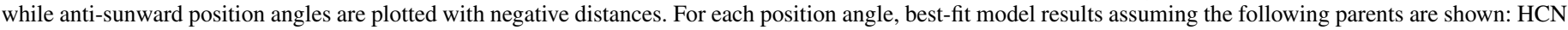
(solid lines), $\mathrm{CH}_{3} \mathrm{CN}$ (dotted lines), and $\mathrm{HC}_{3} \mathrm{~N}$ (dashed lines).

(A color version of this figure is available in the online journal.)

In order to determine further the likelihood that $\mathrm{HCN}$ is the parent of $\mathrm{CN}$ in Encke's coma, we determined the $\mathrm{CN} / \mathrm{OH}$ ratios for each of the sampled position angles using the production rates from the best-fit model results for each observation. Table 2 lists the $\mathrm{CN} / \mathrm{OH}$ ratios and $1 \sigma$ errors obtained for each position angle sampled in all three nights of data. We find an aggregate $\mathrm{CN} / \mathrm{OH}$ ratio of $0.009 \pm 0.004$. Other potential parents, such as $\mathrm{CH}_{3} \mathrm{CN}\left[\beta=6.7 \times 10^{-6}\right.$ (Crovisier 1994)] and $\mathrm{HC}_{3} \mathrm{~N}[\beta=6.6$ $\times 10^{-5}$ (Crovisier 1994)], were also examined with our model. If we assume a sole parent of $\mathrm{CH}_{3} \mathrm{CN}$, we derive an aggregate $\mathrm{CN} / \mathrm{OH}$ ratio of $0.013 \pm 0.007$, and also derive an aggregate $\mathrm{CN} / \mathrm{OH}$ ratio of $0.002 \pm 0.001$ assuming that the sole parent is $\mathrm{HC}_{3} \mathrm{~N}$. The $\mathrm{CN} / \mathrm{OH}$ ratios assuming a sole parent of $\mathrm{HCN}$ obtained here are somewhat higher than the $\mathrm{CN} / \mathrm{OH}$ ratio of 0.003 reported for comet Encke by A'Hearn et al. (1995), but they are on the upper end of the range of their reported $\mathrm{CN} / \mathrm{OH}$ ratios for their comet sample $(0.003 \pm 0.002)$. Their result for Encke is more consistent with our ratio obtained with $\mathrm{HC}_{3} \mathrm{~N}$. However, this may be influenced by differences in the assumed shape of the coma between the two studies, since the A'Hearn et al. study assumed a spherical coma. Therefore, we cannot rule out that $\mathrm{HCN}$ is the sole parent of $\mathrm{CN}$ in Encke.

Future testing of our model will include examinations of the spatial distribution of $\mathrm{NH}, \mathrm{C}_{2}$, and $\mathrm{C}_{3}$, which also produced features in our spectra. Examinations of these other molecules will allow us to look for evidence of nuclear heterogeneity. Because long-slit spectra do not sample the entire coma simultaneously, the morphology of the coma could change significantly over the course of a single night of observations due to the rotation of the nucleus. Investigations of Encke's rotation period by Fernández et al. (2005), Woodney et al. (2007), and Lowry \& Weissman (2008) all suggest a rotation period of $\sim 11 \mathrm{hr}$. One night of 

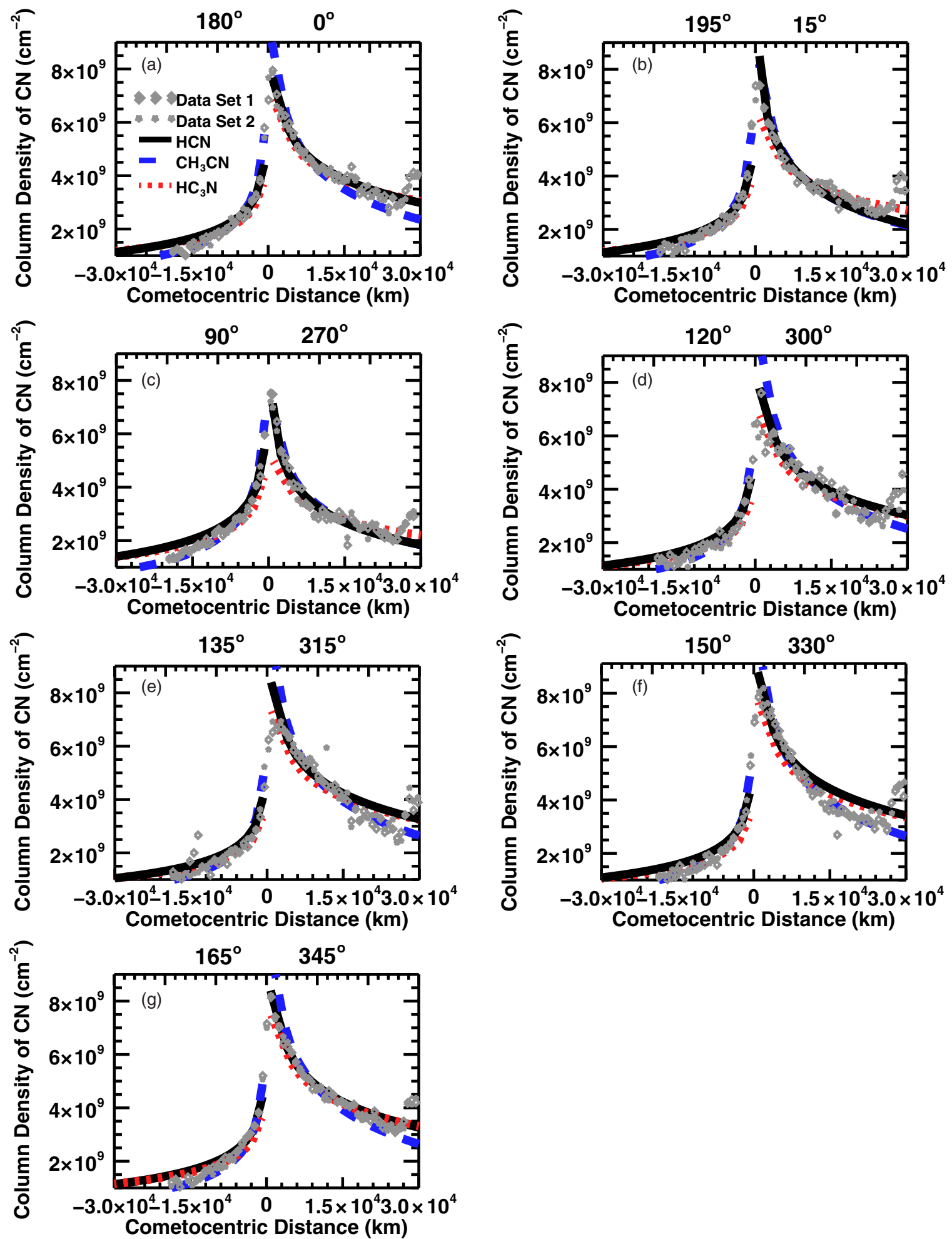

Figure 6. Comparison of best-fit model results with observations of $\mathrm{CN}$ for 2003 October 23 , showing position angles: (a) $0^{\circ}$ and $180^{\circ}$, (b) $15^{\circ}$ and $195^{\circ}$, (c) $90^{\circ}$ and $270^{\circ}$, (d) $120^{\circ}$ and $300^{\circ}$, (e) $135^{\circ}$ and $315^{\circ}$, (f) $150^{\circ}$ and $330^{\circ}$, and (g) $165^{\circ}$ and $345^{\circ}$. In each case, the sampled position angles containing the fan are plotted with positive distances, while anti-sunward position angles are plotted with negative distances. For each position angle, best-fit model results assuming the following parents are shown: $\mathrm{HCN}$ (solid lines), $\mathrm{CH}_{3} \mathrm{CN}$ (dotted lines), and $\mathrm{HC}_{3} \mathrm{~N}$ (dashed lines).

(A color version of this figure is available in the online journal.) 

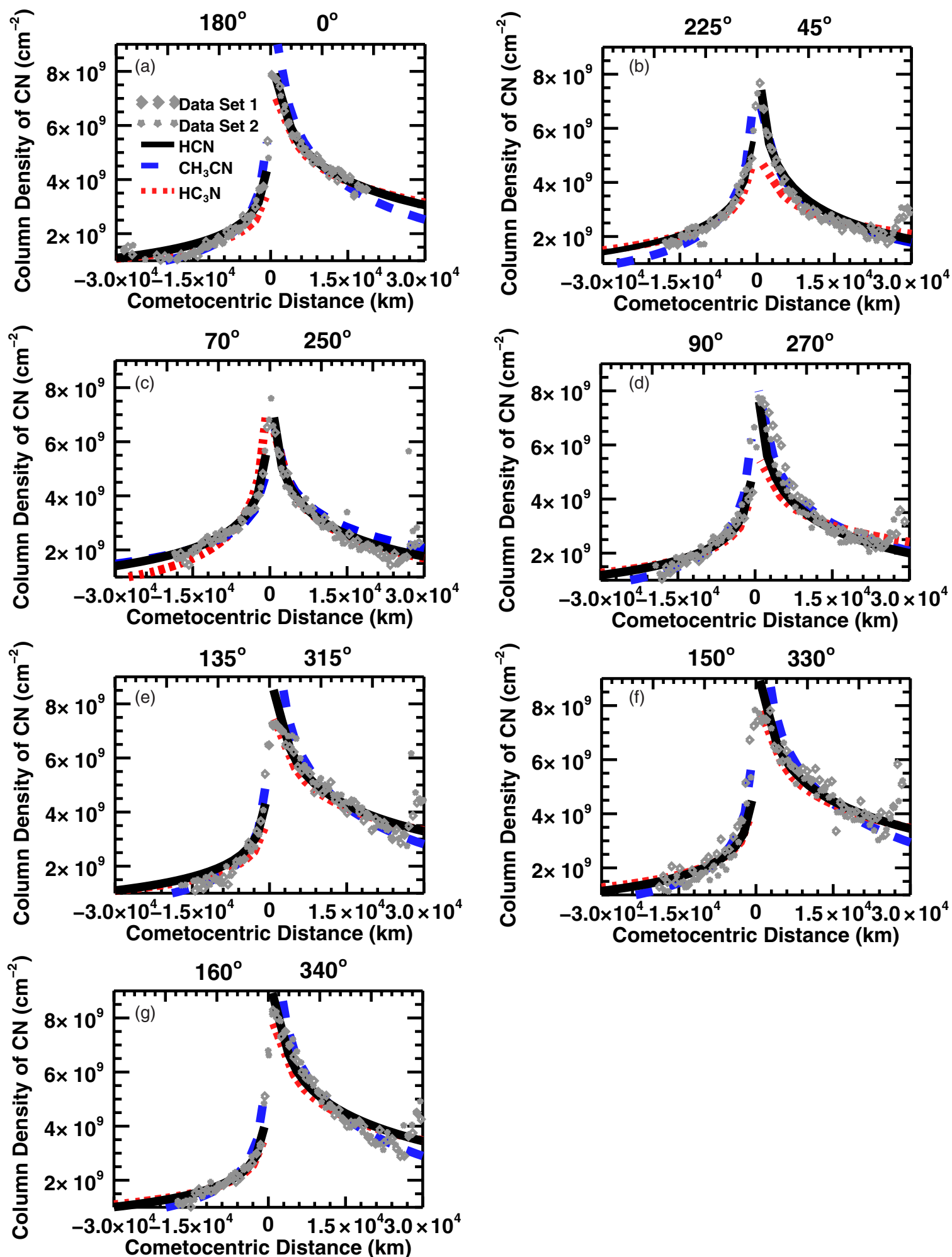

Figure 7. Comparison of best-fit model results with observations of $\mathrm{CN}$ for 2003 October 24 , showing position angles: (a) $0^{\circ}$ and $180^{\circ}$, (b) $45^{\circ}$ and $225^{\circ}$, (c) $70^{\circ}$ and $250^{\circ}$, (d) $90^{\circ}$ and $270^{\circ}$, (e) $135^{\circ}$ and $315^{\circ}$, (f) $150^{\circ}$ and $330^{\circ}$, and (g) $160^{\circ}$ and $340^{\circ}$. In each case, the sampled position angles containing the fan are plotted with positive distances, while anti-sunward position angles are plotted with negative distances. For each position angle, best-fit model results assuming the following parents are shown: $\mathrm{HCN}$ (solid lines), $\mathrm{CH}_{3} \mathrm{CN}$ (dotted lines), and $\mathrm{HC}_{3} \mathrm{~N}$ (dashed lines).

(A color version of this figure is available in the online journal.) 
Table 2

$\mathrm{CN} / \mathrm{OH}$ Ratios $^{\mathrm{a}}$ by Position Angle

\begin{tabular}{|c|c|c|c|c|}
\hline Position Angle & Oct 22 & Oct 23 & Oct 24 & Average \\
\hline $0^{\circ}$ & $0.006 \pm 0.002$ & $0.004 \pm 0.002$ & $0.002 \pm 0.001$ & $0.004 \pm 0.002$ \\
\hline $15^{\circ}$ & $\ldots$ & $0.008 \pm 0.002$ & $\ldots$ & $0.008 \pm 0.002$ \\
\hline $30^{\circ}$ & $0.014 \pm 0.007$ & $\ldots$ & $\ldots$ & $0.014 \pm 0.007$ \\
\hline $45^{\circ}$ & $\ldots$ & $\ldots$ & $0.008 \pm 0.003$ & $0.008 \pm 0.003$ \\
\hline $60^{\circ}$ & $0.029 \pm 0.006$ & $\ldots$ & $\ldots$ & $0.029 \pm 0.006$ \\
\hline $70^{\circ}$ & $\ldots$ & $\ldots$ & $0.004 \pm 0.002$ & $0.004 \pm 0.002$ \\
\hline $90^{\circ}$ & $0.006 \pm 0.004$ & $0.004 \pm 0.004$ & $0.004 \pm 0.002$ & $0.005 \pm 0.004$ \\
\hline $120^{\circ}$ & $0.006 \pm 0.004$ & $0.010 \pm 0.008$ & $\ldots$ & $0.008 \pm 0.006$ \\
\hline $135^{\circ}$ & $\ldots$ & $0.007 \pm 0.004$ & $0.006 \pm 0.002$ & $0.007 \pm 0.003$ \\
\hline $150^{\circ}$ & $0.006 \pm 0.003$ & $0.004 \pm 0.003$ & $0.004 \pm 0.002$ & $0.005 \pm 0.003$ \\
\hline $160^{\circ}$ & $\ldots$ & $\ldots$ & $0.006 \pm 0.003$ & $0.006 \pm 0.003$ \\
\hline $165^{\circ}$ & $\ldots$ & $0.003 \pm 0.002$ & $\ldots$ & $0.003 \pm 0.002$ \\
\hline $180^{\circ}$ & $0.007 \pm 0.003$ & $0.003 \pm 0.002$ & $0.007 \pm 0.003$ & $0.006 \pm 0.003$ \\
\hline $195^{\circ}$ & $\ldots$ & $0.004 \pm 0.002$ & $\ldots$ & $0.004 \pm 0.002$ \\
\hline $210^{\circ}$ & $0.017 \pm 0.004$ & $\ldots$ & $\ldots$ & $0.017 \pm 0.004$ \\
\hline $225^{\circ}$ & $\ldots$ & $\ldots$ & $0.004 \pm 0.002$ & $0.004 \pm 0.002$ \\
\hline $240^{\circ}$ & $0.019 \pm 0.009$ & $\ldots$ & $\ldots$ & $0.019 \pm 0.009$ \\
\hline $250^{\circ}$ & $\ldots$ & $\ldots$ & $0.005 \pm 0.002$ & $0.005 \pm 0.002$ \\
\hline $270^{\circ}$ & $0.010 \pm 0.007$ & $0.006 \pm 0.003$ & $0.005 \pm 0.002$ & $0.007 \pm 0.004$ \\
\hline $300^{\circ}$ & $0.010 \pm 0.006$ & $0.014 \pm 0.004$ & $\ldots$ & $0.012 \pm 0.005$ \\
\hline $315^{\circ}$ & $\ldots$ & $0.009 \pm 0.006$ & $0.014 \pm 0.004$ & $0.012 \pm 0.005$ \\
\hline $330^{\circ}$ & $0.006 \pm 0.003$ & $0.006 \pm 0.004$ & $0.014 \pm 0.007$ & $0.009 \pm 0.005$ \\
\hline $340^{\circ}$ & $\ldots$ & $\cdots$ & $0.007 \pm 0.004$ & $0.007 \pm 0.004$ \\
\hline $345^{\circ}$ & $\ldots$ & $0.004 \pm 0.002$ & $\ldots$ & $0.004 \pm 0.002$ \\
\hline Average & $0.011 \pm 0.004$ & $0.006 \pm 0.003$ & $0.006 \pm 0.003$ & $0.009 \pm 0.004$ \\
\hline
\end{tabular}

Note. ${ }^{a}$ Error bars are $1 \sigma$.

our observations typically occurred over $8 \mathrm{hr}$, which would be nearly three-quarters of the suggested rotation period. Future work will include studies of other comets, such as 4P/Faye, a comet that is also known to have an asymmetric coma (Lamy et al. 1996). These data have been collected with the VIRUS-P integral-field spectrograph (Hill et al. 2008), allowing us to sample the entire coma simultaneously. These observations should serve as an excellent test of the robustness of our model.

\section{CONCLUSIONS}

We have modified the vectorial code in order to analyze $\mathrm{OH}$ and $\mathrm{CN}$ observations of comet Encke. Our modified method of line-of-sight integration through a cone provided reasonable reproductions of the observed $\mathrm{OH}$ and $\mathrm{CN}$ profiles. Furthermore, the $\mathrm{CN} / \mathrm{OH}$ ratios obtained from the model fits to the observations are consistent with values reported elsewhere (A'Hearn et al. 1995; Bockelée-Morvan et al. 2004). As a result, we find that our results are consistent with $\mathrm{HCN}$ being the parent of $\mathrm{CN}$ in comet Encke, but we cannot completely rule out other potential parents such as $\mathrm{CH}_{3} \mathrm{CN}$ or $\mathrm{HC}_{3} \mathrm{~N}$. However, our results do not confirm the two active areas suggested by the results of Sekanina (1988). Instead, our single-cone model is more consistent with the single active area suggested by Woodney et al. (2007).

The authors thank J. P. Morgenthaler and D. Bodewits for providing IDL routines that facilitated our model-building efforts. The authors also thank R. J. Unz for assistance with the code, and M. R. Combi for valuable discussions pertaining to the vectorial model. We also thank the referee for valuable feedback on this paper. This research was supported by NASA's Planetary Atmospheres Program and the Office of the Vice President for Research and Economic Development at Mississippi State
University. This paper includes data taken at the McDonald Observatory of the University of Texas at Austin.

\section{REFERENCES}

A'Hearn, M. F., Hoban, S., Birch, P. V., et al. 1986a, Nature, 324, 649

A'Hearn, M. F., Hoban, S., Birch, P. V., et al. 1986b, ESLAB Symposium on the Exploration of Halley's Comet. Vol. I: Plasma and Gas (ESA Special Publication, Vol. 250; Noordwijk: ESA)

A'Hearn, M. F., Millis, R. L., Schleicher, D. G., Osip, D. J., \& Birch, P. V. 1995, Icarus, 118, 223

A'Hearn, M. F., Schleicher, D. G., Millis, R. L., Feldman, P. D., \& Thompson, D. T. 1984, AJ, 89, 579

Biver, N., Bockelée-Morvan, D., Crovisier, J., et al. 2002, Earth Moon Planets, 90,323

Bockelée-Morvan, D., \& Crovisier, J. 1985, A\&A, 151, 90

Bockelée-Morvan, D., Crovisier, J., Baudry, A., et al. 1984, A\&A, 141, 411

Bockelée-Morvan, D., Crovisier, J., Despois, D., et al. 1987, A\&A, 180, 253

Bockelée-Morvan, D., Crovisier, J., Mumma, M. J., \& Weaver, H. A. 2004, in Comets II, Vol. 745, ed. M. C. Festou, H. U. Keller, \& H. A. Weaver (Tucson, AZ: Univ. Arizona Press), 391

Bonev, B., \& Komitov, B. 2000, BAAS, 32, 1072

Cochran, A. L. 1982, PhD thesis, Univ. Texas

Cochran, A. L., Barker, E. S., Caballero, M. D., \& Györgey-Ries, J. 2009, Icarus, 199,119

Cochran, A. L., Barker, E. S., Ramseyer, T. F., \& Storrs, A. D. 1992, Icarus, 98, 151

Combi, M. R., \& Delsemme, A. H. 1980, ApJ, 237, 641

Crovisier, J. 1994, J. Geophys. Res., 99, 3777

Dello Russo, N., Vervack, R. J., Weaver, H. A., et al. 2009, ApJ, 703, 187

Despois, D., Crovisier, J., Bockelée-Morvan, D., Gerard, E., \& Schraml, J. 1986, A\&A, 160, L11

DiSanti, M. A., \& Mumma, M. J. 2008, Space Sci. Rev., 138, 127

Feldman, P. D., Cochran, A. L., \& Combi, M. R. 2004, in Comets II, ed. M. C. Festou, H. U. Keller, \& H. A. Weaver (Tucson, AZ: Univ. Arizona Press), 425

Fernández, Y. R., Lowry, S. C., Weissman, P. R., et al. 2005, Icarus, 175, 194

Festou, M. C. 1981, A\&A, 95, 69

Festou, M. C., \& Barale, O. 2000, AJ, 119, 3119

Festou, M. C., Barale, O., Davidge, T., et al. 1998, BAAS, 30, 1089 
Fray, N., Bénilan, Y., Cottin, H., Gazeau, M. C., \& Crovisier, J. 2005, Planet. Space Sci., 53, 1243

Gehrz, R. D., Ney, E. P., Piscitelli, J., Rosenthal, E., \& Tokunaga, T. A. 1989, Icarus, 80, 280

Hill, G. J., MacQueen, P. J., Palunas, P., et al. 2008, Proc. SPIE, 7014, 213

Huebner, W. F., Buhl, D., \& Snyder, L. E. 1974, Icarus, 23, 580

Huebner, W. F., Keady, J. J., \& Lyon, S. P. 1992, Ap\&SS, 195, 1

Kim, S. J., A'Hearn, M. F., \& Cochran, W. D. 1989, Icarus, 77, 98

Krasnopolsky, V. A. 1991, A\&A, 245, 310

Lamy, P. L., Toth, I., Grün, E., et al. 1996, Icarus, 119, 370

Lisse, C. M., Fernández, Y. R., A’Hearn, M. F., et al. 2004, Icarus, 171, 444

Lowry, S. C., \& Weissman, P. R. 2008, Icarus, 188, 212

Newburn, R. L., \& Spinrad, H. 1985, AJ, 90, 2591

Reach, W. T., Sykes, M. V., Lien, D., \& Davies, J. K. 2000, Icarus, 148, 80
Schleicher, D. G., \& A'Hearn, M. F. 1988, ApJ, 331, 1058

Schloerb, F. P., Kinzel, W. M., Swade, D. A., \& Irvine, W. M. 1986, ApJ, 310, 55

Schloerb, F. P., Kinzel, W. M., Swade, D. A., \& Irvine, W. M. 1987, A\&A, 187, 475

Sekanina, Z. 1987, Diversity and Similarity of Comets (ESA Special Publication, Vol. 278; Noordwijk: ESA), 315

Sekanina, Z. 1988, AJ, 95, 911

Tatum, J. B. 1984, A\&A, 135, 183

Winnberg, A., Ekelund, L., \& Ekelund, A. 1987, A\&A, 172, 335

Woodney, L. M., A'Hearn, M. F., Schleicher, D. G., et al. 2002, Icarus, 157, 193

Woodney, L. M., Schleicher, D. G., Reetz, K. M., \& Ryan, K. J. 2007, BAAS, 38,486

Ziurys, L. M., Savage, C., Brewster, M. A., et al. 1999, ApJ, 527, 67 